\title{
Transverse galloping of two-dimensional bodies having a rhombic cross-section
}

\author{
D. Ibarra, F. Sorribes, G. Alonso*, J. Meseguer \\ IDR/UPM, E.T.S.I. Aeronáuticos, Universidad Politécnica de Madrid, E-28040 Madrid, Spain
}

\begin{abstract}
Transverse galloping is a type of aeroelastic instability characterized by oscillations perpendicular to wind direction, large amplitude and low frequency, which appears in some elastic two-dimensional bluff bodies when they are subjected to an incident flow, provided that the flow velocity exceeds a threshold critical value. Understanding the galloping phenomenon of different cross-sectional geometries is important in a number of engineering applications: for energy harvesting applications the interest relies on strongly unstable configurations but in other cases the purpose is to avoid this type of aeroelastic phenomenon. In this paper the aim is to analyze the transverse galloping behavior of rhombic bodies to understand, on the one hand, the dependence of the instability with a geometrical parameter such as the relative thickness and, on the other hand, why this cross-section shape, that is generally unstable, shows a small range of relative thickness values where it is stable. Particularly, the non-galloping rhombus-shaped prism's behavior is revised through wind tunnel experiments. The bodies are allowed to freely move perpendicularly to the incoming flow and the amplitude of movement and pressure distributions on the surfaces is measured.
\end{abstract}

\section{Introduction}

Galloping instabilities, either transverse or torsional, have got the attention of many researchers because of their impact on very common engineering problems related to ice accretion on electric transmission lines [1-3], traffic signal gantries and structures [4-6], catenary leads and many other two-dimensional applications. Recently, the interest in the galloping phenomena has increased because of their potential use as a renewable energy source. A large number of papers has been published where the suitability of some specific mechanisms to efficiently harvest electric energy through different energy converters (piezoelectric, electromagnetic, etc.) are considered [7-9]. Therefore, a considerable effort is being devoted to analyze this wind-induced vibration and develop simple theories to evaluate the energy-harvesting capabilities of twodimensional bluff bodies. The main triggering effects of body oscillation can be vortex-induced oscillation, galloping, or wake galloping [10-14].

In the case of galloping, the literature is biased through prone to gallop bodies; a large number of papers dealing with the galloping properties of a wide spectrum of geometries have been published [9,15-18]. Other cases of interest are the twodimensional bodies with highly attenuated oscillations and those which do not gallop. Literature of no-galloping bodies is

\footnotetext{
* Corresponding author. Tel.: +34 913366353; fax: +34913366363.

E-mail address: gustavo.alonso@upm.es (G. Alonso).
} 
limited and scarce, leaving apart some papers of specific three-dimensional geometries [19]. Therefore, the aspect of the identification and characterization of galloping-free bodies, i.e., two-dimensional bodies (different from the cylindrical ones) that do not gallop under a wide range of upstream flow conditions is very interesting from a practical point of view, as in the case of bridges, buildings, road signs, etc.

Concerning transverse galloping instability, a very simple theory like the one due to Den Hartog [20] is enough to elucidate if a given two-dimensional body is suitable to gallop or not. According to this theory, transverse galloping can be explained by taking into account that although the incident wind velocity $U$ is uniform and constant, due to the lateral oscillation of the body, in a body reference system, the total velocity changes both magnitude and direction with time, therefore, the body angle of attack also changes with time, hence there are aerodynamic forces acting on the body.

In the simplest model of galloping (one degree of freedom model) it is assumed that the two-dimensional body, whose mass per unit length is $m$, is elastically mounted on a support with a damping coefficient $\zeta$ and a stiffness $m \omega^{2}$ (where $\omega$ is the angular natural frequency). Within this approximation, if the aerodynamic force (proportional in this case to $\mathrm{d} z / \mathrm{d} t$ ) is considered as a contribution to the total damping of the system, the total damping coefficient is

$$
\zeta_{T}=\zeta+\frac{\rho U b}{4 m \omega}\left(\frac{\mathrm{d} c_{l}}{\mathrm{~d} \alpha}+c_{d}\right),
$$

where $\rho$ is the fluid density, $b$ is the characteristic length, $c_{l}$ is the lift coefficient and $c_{d}$ is the drag coefficient, both dependent on the angle of attack, $\alpha$. The oscillation will be stable if $\zeta_{T}>0$ and unstable if $\zeta_{T}<0$. As the mechanical damping $\zeta$ is generally positive, instability will only occur if

$$
H=\frac{\mathrm{d} c_{l}(\alpha)}{\mathrm{d} \alpha}+c_{d}(\alpha)<0
$$

expression known as the Den Hartog criterion. Hence, the sufficient condition for galloping is $\zeta_{T}<0$, or, according to Eq. (1):

$$
\frac{\mathrm{d} c_{l}}{\mathrm{~d} \alpha}+c_{d}<-\frac{4 m \zeta \omega}{\rho U b}
$$

It must be pointed out that in Eq. (3) the second member tends to zero when the wind velocity increases, which means that the possibility of galloping becomes higher as the wind velocity increases.

From the inspection of Eq. (1), since the aerodynamic drag coefficient is positive, it is clear that the slope of the aerodynamic lift coefficient versus angle of attack curve must be negative, i.e., the body must be stalled $\left(\mathrm{d} c_{l}(\alpha) / \mathrm{d} \alpha<0\right)$ and the absolute value of this slope curve must be larger than the drag coefficient. Implicit in the derivation of this galloping criterion is the hypothesis of quasi-static movement (oscillations are slow enough compared to the incident flow velocity).

There are many two-dimensional bodies which meet this condition for galloping, but the Den Hartog criterion is not able to predict the galloping stability of a given geometry unless the aerodynamic coefficients are determined externally. For bluff bodies the most practical way of determining the aerodynamic coefficients is by means of wind tunnel tests [20]. Once the generic body shape is fixed (identified by some characteristic parameter like relative thickness), galloping instability regions can be drawn in the geometrical parameter versus angle of attack plane; from this, the different regions where the configuration is stable or unstable can be identified.

Such analysis has been performed at IDR/UPM experimental facilities and the galloping properties of different families of two-dimensional bodies with different cross-section shapes have been published (triangles, rhombi, biconvex, elliptical, rectangles, etc. [15-17]). These bodies show transverse galloping instability in certain ranges of the angle of attack, and they are stable outside these specific limits. Leaving apart circular cylinders (they do not gallop because $\mathrm{d} c_{l} / \mathrm{d} \alpha=0$ ) there is a case where bodies show a free-of-galloping behavior no matter what the value of the angle of attack: this is the case of rhombusshaped prisms having a value of their relative thickness close to 0.36 [17].

According to data published in [17] there is a gap spanning between $\tau \cong 0.36$ and $\tau \cong 0.40$ ( $\tau$ is the relative thickness, defined as the ratio of the rhombus thickness, $t$, to its chord, $c, \tau=t / c$, Fig. 1) where no galloping was observed in the whole range of angles of attack $(0 \leq \alpha \leq \pi / 2)$. Those results were obtained by measuring the lift and drag coefficients through static wind tunnel tests, and then by applying the Den Hartog criterion (Eq. (2)).

Measured results seem to indicate that no-galloping episodes occur in the full range of angles of attack covered in the experimental tests $(0 \leq \alpha \leq \pi / 4)$. No larger values of the angle of attack have been considered since outside this range of interest the large values of the aerodynamic drag prevents the appearance of galloping phenomena. For practical applications, it would be of great interest to know if this kind of geometry does not gallop, independently of the angle of attack at which it is collocated.

As it has already been mentioned, wind tunnel static tests to determine aerodynamic coefficients and therefore applying the quasi-static Den Hartog criterion for galloping were already performed [17]. This is an indirect way of assessing the galloping instability of a given geometry. The quasi-steady Den Hartog criterion for galloping is a first attempt to clarify if a given body section can gallop or not. This is a necessary condition, but from the dynamic approach there are other factors affecting the galloping behavior, i.e., those related to the mechanical characteristics of the oscillating body (damping, stiffness), as well as those of the incoming flow (mainly turbulence).

What the present work aims at verifying is, in the first place, the suitability of the quasi-static criterion for this type of bodies. In order to do that, dynamic wind tunnel tests have been performed. That means that the bodies are supported 
(a)

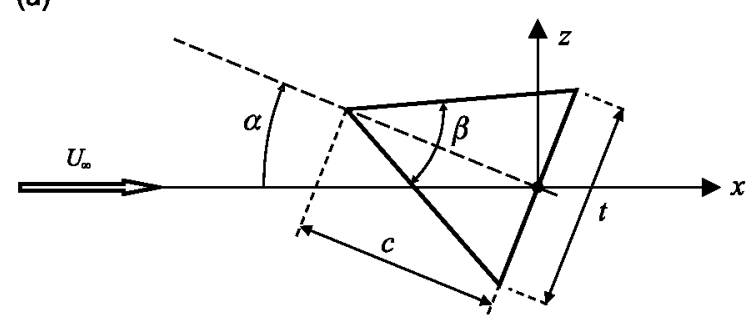

(b)

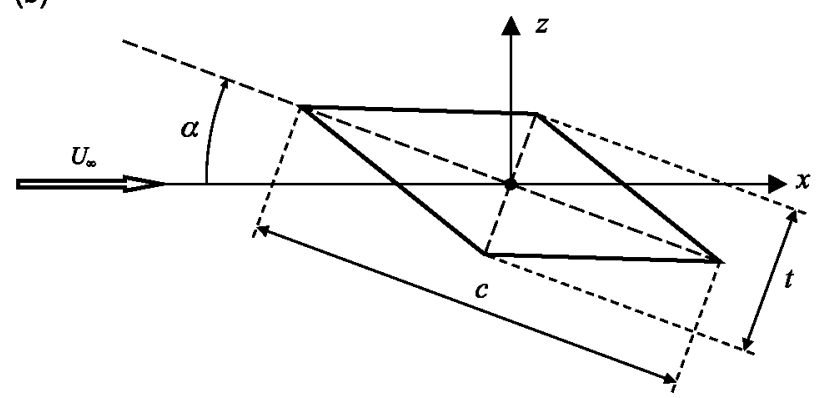

Fig. 1. Sketch of a triangular (A) and a rhombic (B) cross-section bodies with the parameters that identify the different configurations: angle of attack, $\alpha$; main vertex angle, $\beta$; chord, $c$; and maximum thickness, $t$.

elastically in the wind tunnel and therefore can oscillate freely under the action of the incident flow. A direct corroboration of the galloping stability has been performed by measuring directly oscillations and observing how those oscillations grow (or do not) with the incident velocity. Formally speaking, galloping is a type of oscillation that appears once the flow velocity becomes greater than a given threshold velocity, provided that the body section shape is prone to gallop. Once that threshold velocity is reached, the amplitude of the limit cycle oscillation increases very rapidly with the flow velocity, normally until the structure collapses (in real applications). Galloping is a destructive phenomenon, in such a way that when galloping is experimentally studied, some limitation on the oscillation amplitude must be implemented in the experimental facility to avoid the destruction of both, the model under analysis and the facility. Of course, a given body section can oscillate due to the aerodynamic interaction with the incoming flow, but this is not dangerous for the body integrity, since from the point of view of the body dynamics the oscillations remain limited within a small range (something similar happens with vortex-shedding oscillations), and those oscillations are not related to galloping (i.e., there is not a threshold velocity from where the amplitude of the oscillations increases rapidly with the flow velocity).

In the second place, the present work aims at understanding the phenomenon by exploring the flow morphology around the bodies through the measurement of the pressure distribution, because it is well known that the flow morphology on the body surfaces explains the galloping instability, and the main purpose of the present work is to investigate why only a small group of rhombi (classified according to the relative thickness) is stable to transverse galloping while the rest of the relative thicknesses is unstable.

In the next section the experimental facilities are described. Experimental results are presented and analyzed in Section 3 and, finally, conclusions and remarks are outlined in Section 4.

\section{Experimental setup and procedures}

All the measurements were done in the $\mathrm{A} 4 \mathrm{C}$ wind tunnel, an open circuit wind tunnel located at IDR/UPM. It has a $0.20 \mathrm{~m}$ width, $1.80 \mathrm{~m}$ height and $2.00 \mathrm{~m}$ long test section. The upstream turbulence intensity can be modified by changing the porosity of a wire mesh which is placed at the tunnel's contraction entrance. The dynamic pressure in the test section is measured by an Air Flow 048 Pitot tube connected to a Druck LPM5480 pressure transducer. Two types of aerodynamic tests were performed: free transverse oscillation amplitude measurements and pressure measurements.

For the free oscillation tests (one degree of freedom), the wind tunnel is equipped with an external device (Fig. 2) consisting of two parallel cylindrical shafts with air bushings which support a rigid platform. Such a platform is anchored to a rigid frame through springs. In turn the platform supports the model through a shaft perpendicular to the platform. Since the shaft is locked, and it cannot rotate, this mechanism allows only the vertical oscillation of the model inside the test section and any other motion is locked. In addition, because of the air bushings, most of the friction is eliminated. The vertical displacement of the body is measured with a laser distance sensor M7L (100 mm range) from MEL. The zero offset point was in the middle of the measuring range, allowing to measure oscillations of $\pm 50 \mathrm{~mm}$.

During the tests no special provisions were undertaken to avoid air flow through the gap $0.5 \mathrm{~cm}$ long between the model and the wind-tunnel test section walls, this gap has not been taken into account to correct the measured results (in any case 
(a)

(b)

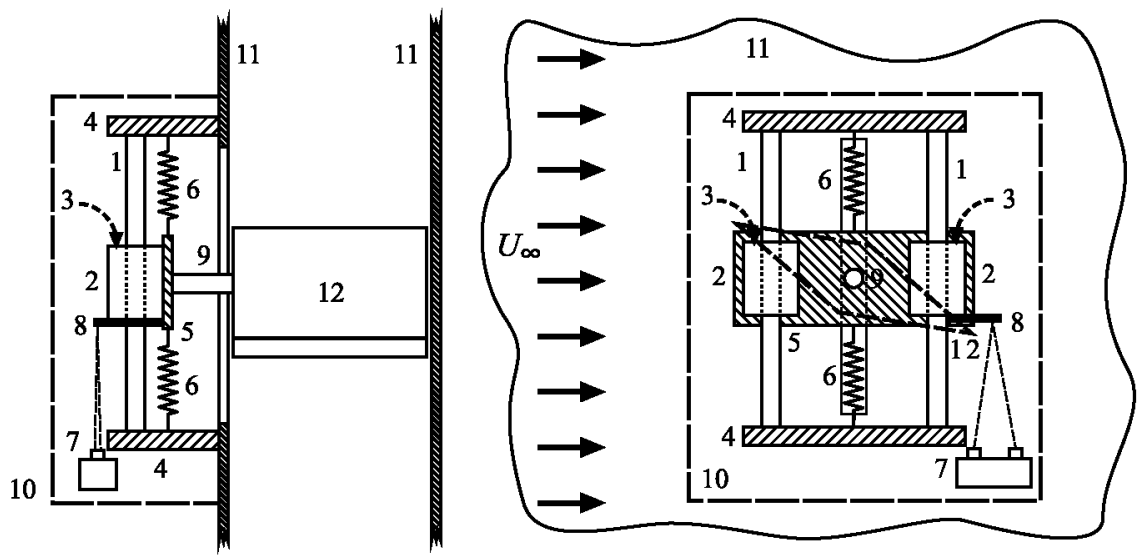

Fig. 2. Sketch of the apparatus used for free vibration tests. (A) Front view, (B) lateral view. The labels identify the different components according to the following key: (1) cylindrical guides, (2) air bushings, (3) air supply, (4) oscillation mechanism frame, (5) rear air bushing frame, (6) springs, (7) M7L/100 laser distance sensor, (8) laser mirror, (9) rotating shaft, (10) airtight section, (11) wind tunnel walls, (12) test section.

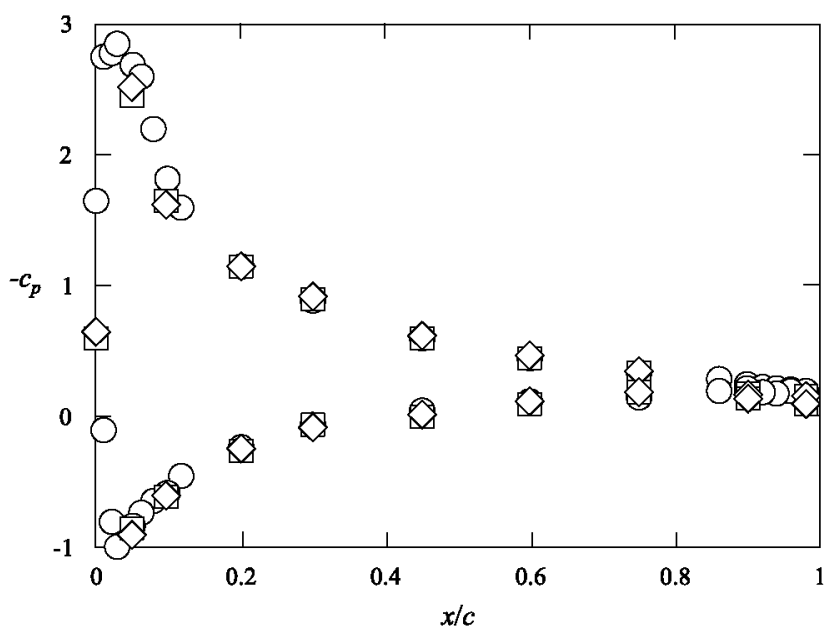

Fig. 3. Pressure distributions measured on an NACA 0012 aerofoil in the A4C wind tunnel. The symbols identify the measurement section according to the following key: $y / d=1 / 9$ (rhombi), $1 / 4$ (squares) and $1 / 2$ (circles), where $y$ is the distance from the model tip and $d$ the model span.

its effect is assumed to be small as is explained below). In order to verify that this assumption is correct, the characterization procedure of all two-dimensional wind tunnels existing at IDR/UPM includes some previous measurements by using a symmetrical aerofoil model equipped with pressure taps. In the case of the A4C wind tunnel the model is an NACA 0012 [21], equipped with 57 pressure taps distributed in three sections, located at $y / d=1 / 9,1 / 4$ and $1 / 2$, where $y$ is the distance from one of the model tips and $d$ is the model span $(d=19.5 \mathrm{~cm})$. At each measuring section there are 9 pressure taps on the upper surface, another 9 pressure taps on the lower surface, plus one additional pressure tap at the leading edge. In these previous tests, pressure distributions were measured, and recorded, at $125 \mathrm{~Hz}$ sampling rate during $10 \mathrm{~s}$. This test was used to evaluate the two-dimensional character of the flow acting on the aerofoil, as it was demonstrated from the comparison of measured pressure distributions at different span locations, where no significant differences were found (Fig. 3).

In the free oscillation tests two types of prisms were tested: triangular and rhombic cross section bodies. All the models used in the free vibration tests are made of wood, by using an isotropic artificial wood $5 \mathrm{~mm}$ in thickness for the lateral sides, and plywood of several layers, $1.2 \mathrm{~mm}$ thick, for prism surfaces; the mass per unit length is similar in all of the tested models (Table 1).

Three triangular cross-section prisms and three rhombic cross section bodies were tested. The physical properties of these test samples are listed in Table 1 . The test procedure was the following: once the wind tunnel is set at the selected velocity and after waiting for $60 \mathrm{~s}$ for flow stabilization, the data acquisition system records the laser distance sensor output at $200 \mathrm{~Hz}$ sampling rate during $30 \mathrm{~s}$; then the wind tunnel velocity is varied by a small step and a new measurement period starts. In the case of triangular bodies, the experimental phase of increasing velocity must be stopped when the translational 
Table 1

Physical properties of tested bodies. Triangle main vertex angle, $\beta$; maximum rhombus relative thickness, $\tau$; mass per unit length, $m$; characteristic length, $c$ (distance from the main vertex to the opposite side in triangular bodies, or chord in rhombic prisms); and first natural frequency, $\omega_{n}$.

\begin{tabular}{llll}
\hline $\begin{array}{l}\text { Triangular cross-section } \\
\beta[\mathrm{deg}]\end{array}$ & $m[\mathrm{~kg} / \mathrm{m}]$ & $c[\mathrm{~m}]$ & $\omega_{n}[\mathrm{~Hz}]$ \\
\hline 55 & 6.23 & 0.120 & 0.117 \\
60 & 6.30 & 0.114 & 2.64 \\
65 & 6.28 & $c[\mathrm{~m}]$ \\
Rhombic cross-section & & 2.63 \\
$\tau$ & $m[\mathrm{~kg} / \mathrm{m}]$ & 0.248 \\
\hline 0.36 & 6.63 & 0.248 \\
0.40 & 7.03 & 0.248 \\
0.50 & 7.29 & 2.56 \\
\hline
\end{tabular}

oscillation amplitude reaches the maximum displacement allowed by the experimental set-up, and then the decreasing velocity phase is started.

Air flow velocity inside the wind tunnel test section is controlled through the frequency of the AC current supplied to the electric motors that power the wind tunnel (the dependence between both parameters is linear provided that the power supply frequency is larger than $10 \mathrm{~Hz}$ ). In all cases this frequency was increased at $2 \mathrm{~Hz}$ steps from $0 \mathrm{~Hz}$ to $10 \mathrm{~Hz}$, and then at $1 \mathrm{~Hz}$ step from $10 \mathrm{~Hz}$ to the frequency that corresponds to the maximum flow velocity, which is $26 \mathrm{~Hz}$ in the case of triangular bodies and $36 \mathrm{~Hz}$ in the case of rhombi. It must be pointed out that the reduced velocity is double in the triangles due to the fact that its characteristic length is half of that of the rhombi (see Table 1), i.e., taking into account that the natural frequencies of all the tested bodies are almost equal, in the linear frequency/air-velocity regime a $1 \mathrm{~Hz}$ step in frequency corresponds to a reduced velocity step close to 1.4 in rhombi, and close to 2.8 in triangular bodies.

From Pitot tube records the average air speed, $U$, was calculated, as well as the reduced velocity, $U_{r}=U /\left(\omega_{n} c\right)$, where $\omega_{n}$ is the first natural frequency and $c$ the characteristic length (both defined in Table 1), and from the laser distance sensor, records of the dimensionless root mean square of the lateral displacement $A=a_{\mathrm{RMS}} / c$ are obtained, where $a_{\mathrm{RMS}}$ is the root mean square value of the dimensional amplitude series $a(t)$ and $c$ is the characteristic length defined in Fig. 1 .

For pressure measurements a rhombus model with $c=294 \mathrm{~mm}, \tau=0.36$, and span $190 \mathrm{~mm}$ has been made. The surfaces are made out of a steel plate $0.5 \mathrm{~mm}$ thick; as these measurements are static, the mass of the model is irrelevant. On each side of the rhombus there are 15 pressure taps ( 60 total) distributed in the middle section of the body; they are spaced $10 \mathrm{~mm}$ between them, starting at $15 \mathrm{~mm}$ from the leading edge and up to $15 \mathrm{~mm}$ to the trailing edge. Pressure taps are made out of a brass tube that is $30 \mathrm{~mm}$ long and has $1 \mathrm{~mm}$ internal diameter; the tube is mounted such that one of its ends is carefully positioned flush with the surface where pressure is to be measured, whereas the opposite end is connected through a flexible plastic tube to the measurement equipment (pressure taps are connected to a pressure scanner, model ZOC 33/64PxX2 from Scanivalve) which is placed outside the wind tunnel test section. The model is mounted on an ESP100/RV80PP rotating platform from Newport. Both the pressure scanner and the rotating platform are placed inside a tight chamber located adjacent to the tests section. All the experimental sequences, the different values of the angle of attack, pressure measurements and data saving, are controlled by a PC. The pressure distributions were recorded at $200 \mathrm{~Hz}$ sampling rate during $30 \mathrm{~s}$.

\section{Experimental results}

Since triangular bodies show a marked galloping behavior [15,22], they were used as a reference for comparison with rhomboidal ones. The results concerning triangular bodies are shown in Fig. 4, where the variation of the dimensionless root mean square oscillation amplitude with the reduced velocity is drawn. Some of the results reported here can be compared with those published in $[15,22]$ however, there exist some differences due to the oscillation mechanism which, as stated earlier, minimizes the friction effects.

It is known that if the system is found to be unstable, it means that the amplitude will begin to grow; how far this growing will develop depends on the nature of the nonlinearity [20]. According to Fig. 4, triangular prims with a main vertex angle close to $\beta=60^{\circ}$ show transverse galloping instability at angles of attack close to $\alpha=180^{\circ}$. In a defined geometry (i.e., the results observed in each column), the angle of attack determines not only the starting velocity of galloping, but also the amplitude of oscillation. In the case $\alpha=180^{\circ}$, the tendency of growing would lead the body to an oscillation amplitude outside the measuring range (from this fact the unstable behavior of the body is clearly deduced). Thus, the increasing wind velocity phase ends when the oscillation amplitude of a given body approaches the maximum amplitude allowed by the oscillation mechanism. After that point, the incident flow velocity is decreased and a hysteresis cycle either appears or it does not depending on the value of the angle of attack as well as on the existence of inflection points in the $c_{l}$ versus $\alpha$ curve. Also, it is possible to see that triangular bodies show transverse galloping instability at large reduced wind velocities. 

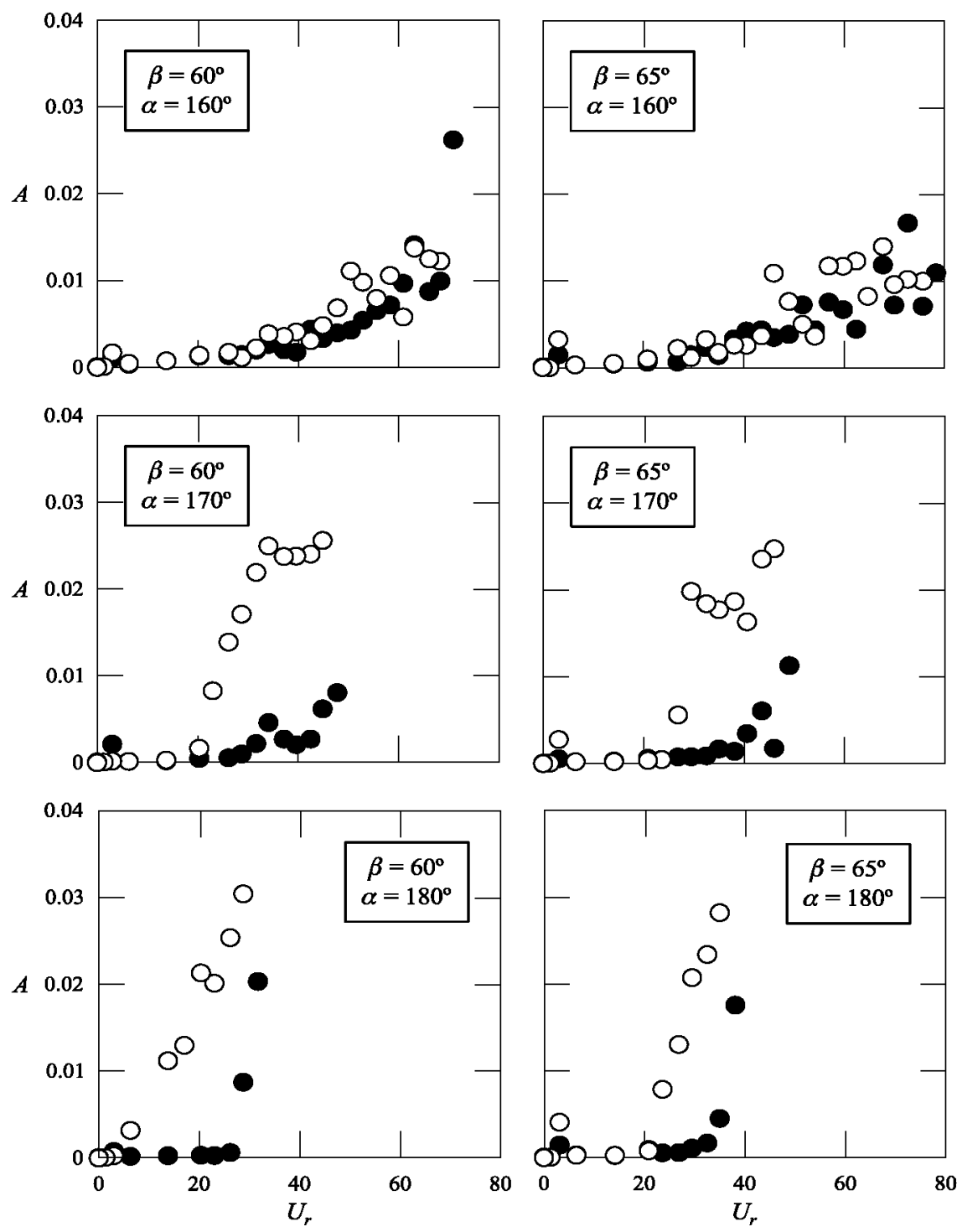

Fig. 4. Variation with the reduced velocity, $U_{r}$, of the dimensionless root mean square oscillation amplitude, $A$. Experimental results correspond to triangular cross-section bodies with main vertex angles $\beta=60^{\circ}$ and $\beta=65^{\circ}$, at angles of attack $\alpha=160^{\circ}, \alpha=170^{\circ}$ and $\alpha=180^{\circ}$. Black circles indicate results measured in the increasing reduced velocity phase, whereas white ones indicate results obtained while decreasing reduced velocity.

In comparison with the results reported in [22], there are some differences due to the oscillation device; in that case larger friction effects are involved and the wind velocity has to be larger than some threshold value to trigger the body oscillation, a typical value being $U_{r}=20$. However, with the air bushing mechanism, the translational body motion can be detected from very small values of the flow velocity. This explains the appearance of a small dimensionless amplitude peak at small reduced velocities $\left(U_{r} \cong 4\right.$ ), which corresponds to a vortex-induced vibration episode (Fig. 4) in which oscillations are excited by the vortices shed into the body wake (vortex-induced vibrations, VIV). It must be highlighted that a similar VIV behavior has not been detected in the case of rhombic prisms. The results obtained for the case of rhombi are depicted in Fig. 5 (although tests were performed by changing the angle of attack from $0^{\circ}$ to $40^{\circ}$ at $5^{\circ}$ steps, in this plot only the results corresponding to angles of attack close to $\alpha=25^{\circ}$, where galloping instability could occur, have been represented).

The rhombi behaviors shown in Fig. 5 are corroborated by applying the Den Hartog criterion for galloping: in the case of rhombic prisms there is a range of relative thicknesses around $\tau=0.38$ where galloping instability does not appear [17]. As the reduced velocity $U_{r}$ increases, the rhombic oscillation amplitude slightly increases until a maximum value of the dimensionless RMS amplitude $A$ is reached and beyond this critical reduced velocity, further increments of the flow velocity imply the decrease of the oscillation amplitude. In these cases, vortex-induced vibration episodes have not been detected either. This is no longer valid in the cases for $\alpha=25^{\circ}$ and $\alpha=30^{\circ}$ with $\tau=0.50$, where the oscillation amplitude is clearly built up, these two configurations being unstable (see Fig. 6). The stability map shown in Fig. 6, taken from [17], corresponds to 

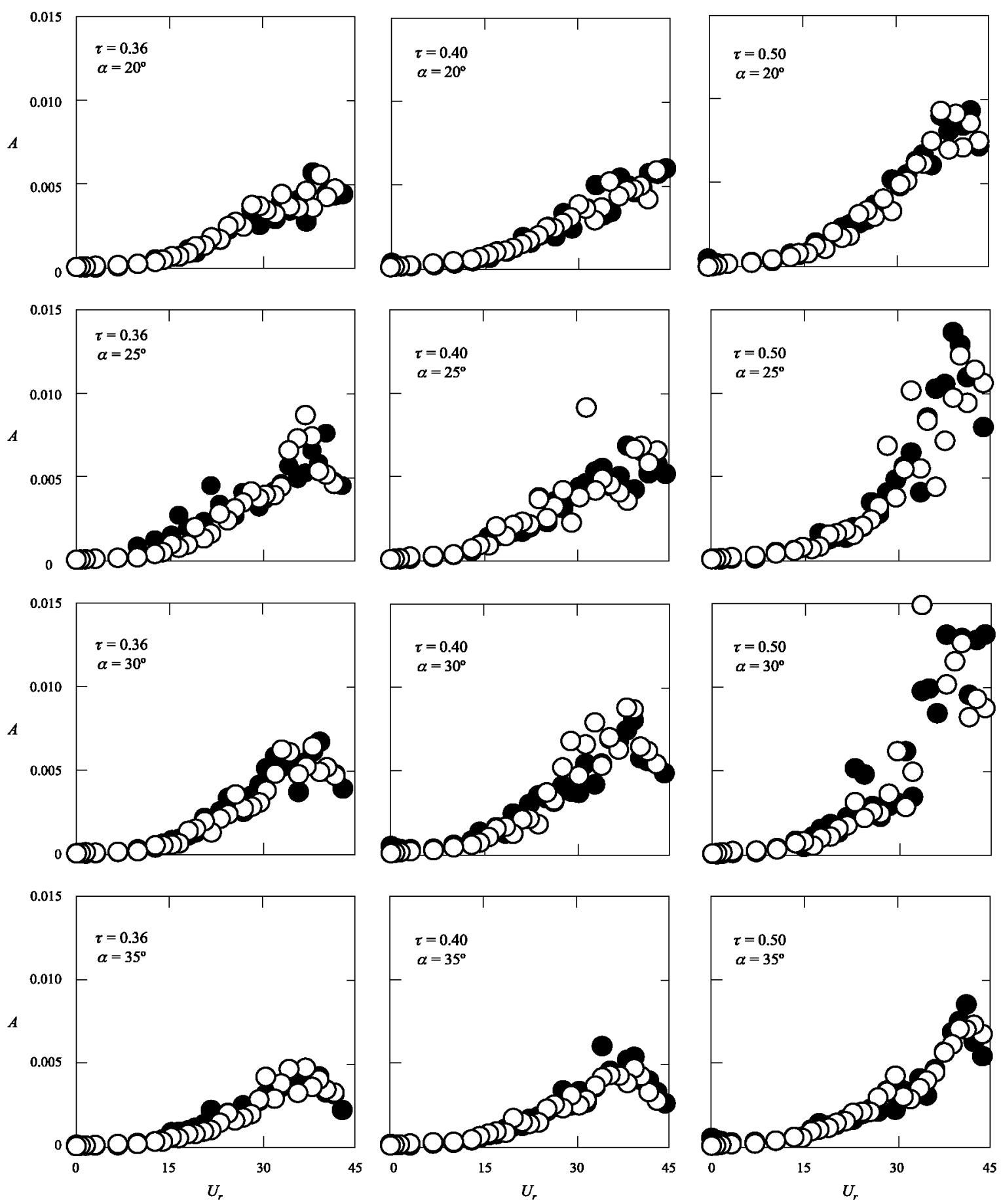

Fig. 5. Variation with the reduced velocity, $U_{r}$, of the dimensionless root mean square oscillation amplitude, $A$. Experimental results correspond to rhombic bodies with different relative thicknesses $(\tau=0.36, \tau=0.40$, and $\tau=0.50)$, at angles of attack ranging from $\alpha=20^{\circ}$ to $\alpha=35^{\circ}$, at $5^{\circ}$ steps. Black circles indicate results measured in the increasing reduced velocity phase, whereas white ones indicate results obtained while decreasing reduced velocity.

the application of the quasi-static galloping criterion to rhombi, and it shows regions of stability/instability, in an angle of attack - relative thickness plane, i.e., which geometries are unstable and at which angle of attack.

Having in mind the stability map that is depicted in Fig. 6 [17], rhombic bodies show galloping instability at very small values of the angle of attack, i.e., $\alpha<6^{\circ}$, for relative thickness around $\tau=0.30$ (say $0.25 \leq \tau \leq 0.34$ ), and they are also unstable in a narrow band spanning from $8^{\circ} \leq \alpha \leq 15^{\circ}$ at $\tau=0$ to $25^{\circ} \leq \alpha \leq 26^{\circ}$ at $\tau \cong 0.33$. As $\tau$ increases there is a zone free of galloping and the galloping instability appears again as long as the relative thickness becomes larger than $\tau \cong 0.42$. 
The rather different behavior of triangles and rhombi is compared in Fig. 7, where experimental results of three different configurations are shown: a rhombic body $\left(\tau=0.40, \alpha=30^{\circ}\right)$, which is a stable configuration and two unstable triangular bodies' configurations $\left(\beta=60^{\circ}, \alpha=175^{\circ}\right.$ and $\left.\beta=55^{\circ}, \alpha=170^{\circ}\right)$.

When comparing the different families of experimental points represented in Fig. 7 it must be taken into account that, since triangular bodies are unstable, their oscillation amplitude became out of range, but with a clear tendency to build up so that the experimental sequence was aborted when the oscillation amplitude became large enough (around 3 times the maximum amplitude of the rhombic body). However, rhombus amplitudes remain within certain limits independently of the relative thickness and angles of attack (provided that the configuration lies in the stable region as shown in Fig. 6).

An explanation of why the rhombic bodies do not show galloping instability in the range of $0.36 \leq \tau \leq 0.40$, regardless of the values of the angle of attack, can be obtained from the pressure distribution on the rhombus surfaces as well as from the total aerodynamic forces acting on the whole prism, as discussed below.

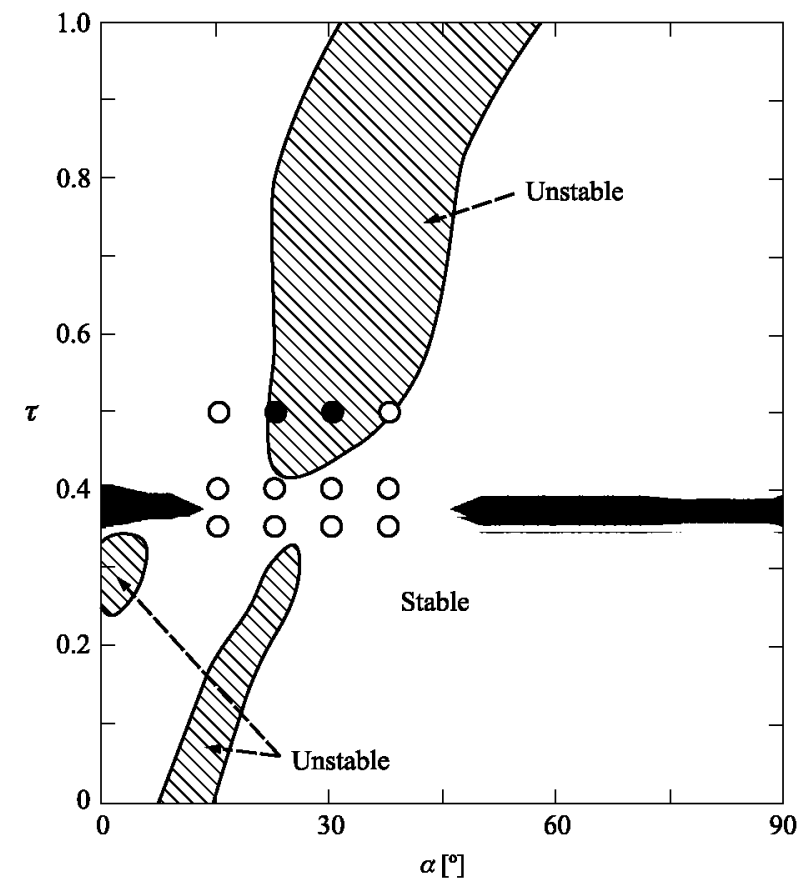

Fig. 6. Stability map of rhombic bodies in the relative thickness versus angle of attack plane $(\tau-\alpha)$ [17]. The shadowed area around $\tau=0.38$ represents the region where no galloping instability has been detected. Circles indicate the tested configurations, with indication of the type of response: stable (white symbols) or unstable (black symbols).

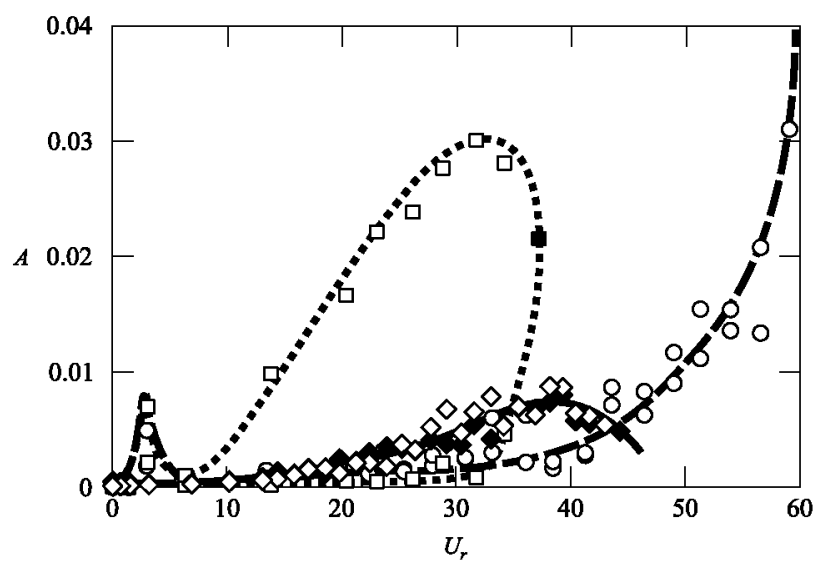

Fig. 7. Variation with the reduced velocity, $U_{r}$, of the dimensionless root mean square oscillation amplitude, $A$, of several oscillating two-dimensional bodies. Rhombi: rhombic bodies (continuous line, $\tau=0.40, \alpha=30^{\circ}$ ); squares: triangular bodies (dotted line, $\beta=60^{\circ}, \alpha=175^{\circ}$ ); and circles: triangular bodies (dashed line, $\beta=55^{\circ}, \alpha=170^{\circ}$ ). Dark symbols, either black or gray, indicate results measured in the increasing reduced velocity phase, whereas white ones indicate results obtained while decreasing reduced velocity. 
Since rhombus body surfaces are flat, total aerodynamic forces can be easily obtained from the pressure distribution measured by considering the pressure forces acting on each one of the four surfaces that form the rhombus (although these are not the only forces acting on the body, and a suction peak acting on the sharp leading edge should also be considered, as well as friction forces, although the last ones are negligible when boundary layer is separated). The fulfilment of the Den Hartog criterion for galloping implies the stalling of the oscillation body, that is, $\mathrm{d} c_{l} / \mathrm{d} \alpha<0$. However, as it can be observed in Fig. 8, pressure distributions on the lower surfaces remain almost the same in the range of angles of attack of interest, whereas in the upper surfaces there is still a recirculation bubble at the leading edge (leading upper surface), while boundary layer is completely separated at the trailing upper surface. This configuration remains until the bubble reattachment point reaches the wedge formed by both upper surfaces, and beyond this angle of attack the boundary layer becomes fully separated. The variation of the lift force with the angle of attack shows a positive slope (Fig. 9), which prevents the appearance of galloping phenomena. It must be stressed that depending on the experimental facility this slope can be slightly negative (as it was reported in [17], depending basically on the incident flow turbulence), but at the same time the drag coefficient is large so that $\mathrm{d} c_{l} / \mathrm{d} \alpha+c_{d}>0$.

Analyzing further the flow morphology around the body, which is known to be responsible for the stability/instability of a given configuration, from Fig. 8 a recirculation bubble can be appreciated at the upper leading edge of the rhombi. Then, as the angle of attack increases, it is possible to appreciate the displacement downstream of the reattachment point up to $\alpha=30^{\circ}$, when the flow does not find any more body to reattach and it is appreciated completely detached. Rhombi with smaller aspect ratio (less than 0.36 ) behave very similarly to a flat plate at around $\alpha=8^{\circ}$, the angle at which it stalls.
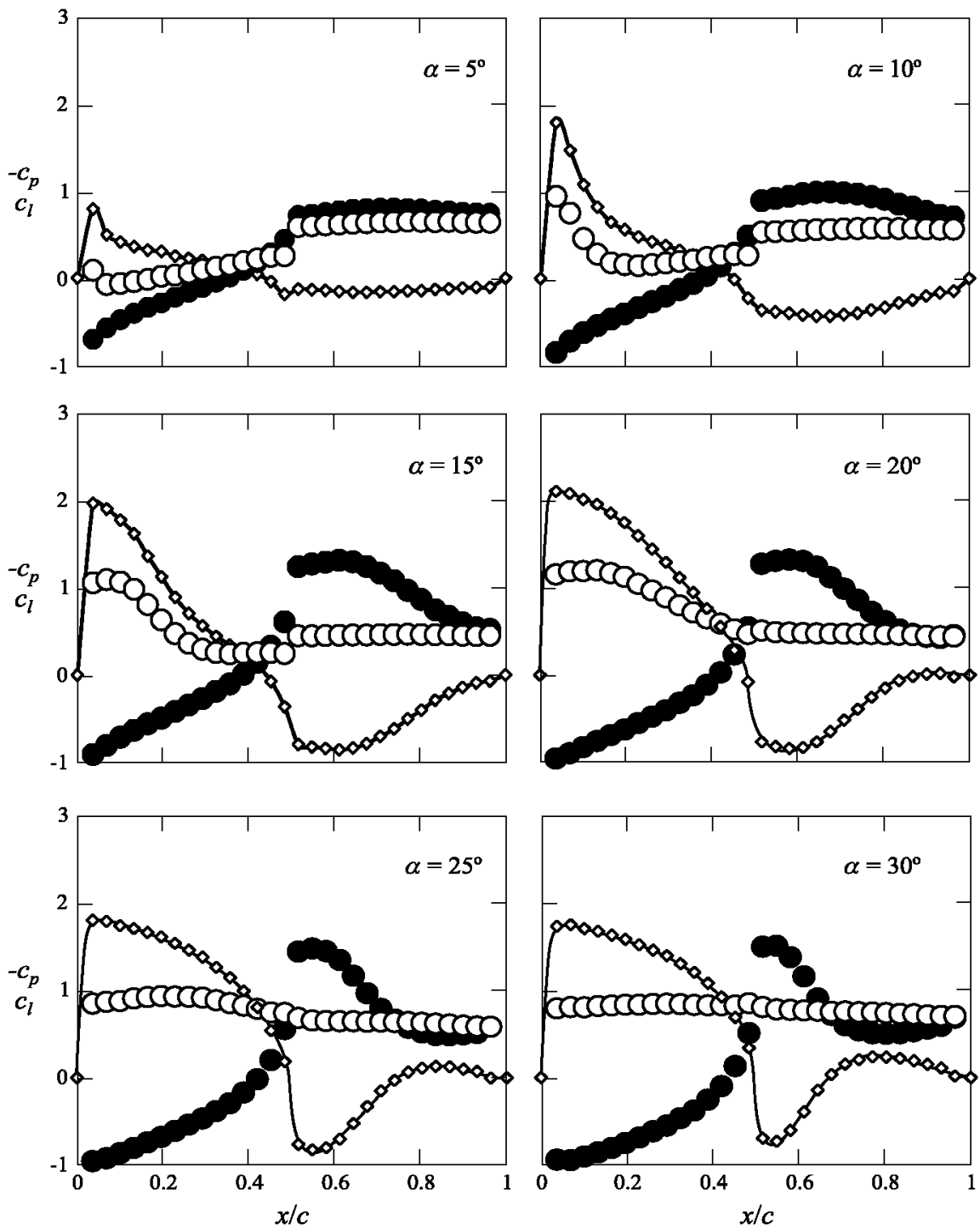

Fig. 8. Pressure coefficient distribution, $c_{p}$, on the rhombus upper surface (white circles) and on the lower surface (black circles) and lift coefficient distributions, $c_{l}$, (small white rhombi) at different angles of attack $\alpha$. 


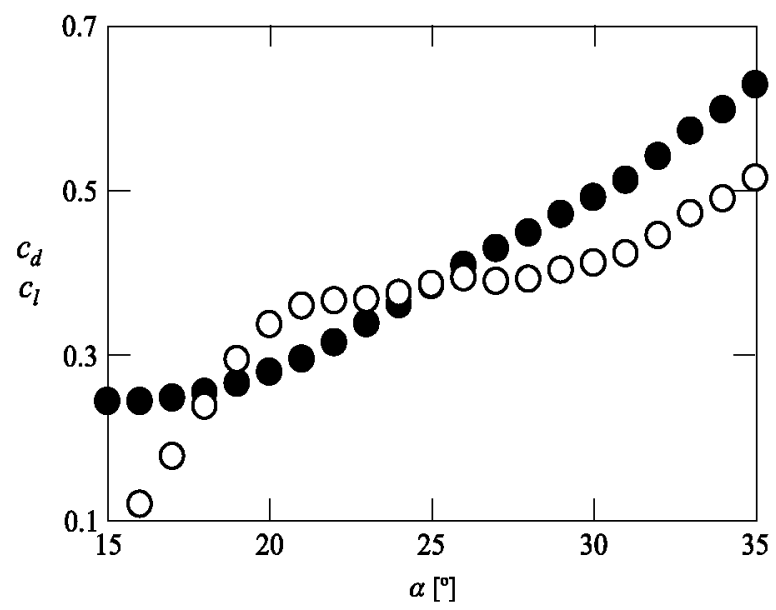

Fig. 9. Variation of the lift coefficient, $c_{l}$ (white symbols) and drag coefficient, $c_{d}$ (black symbols) with the angle of attack, $\alpha$, of rhombic prisms with relative thickness $\tau=0.36$.

The recirculation bubble reattaches the flow behind the vertex in the middle of the chord, in the upper surface, resulting in an increment of the pitch moment when the bubble disappears at around $\alpha=20^{\circ}$. In the rhombi with an aspect ratio around 0.36 the bubble cannot reattach like in the previous case, causing a smaller decrease in the lift coefficient and preventing the appearance of galloping (that depends on the negative slope of the lift coefficient curve). For bigger aspect ratios, larger than 0.46 , antisymmetrical behavior starts to appear near the inflection point in the lift coefficient curve, close to $\alpha=30^{\circ}$; this point moves to higher angles of attack as the aspect ratio increases following the angle between the surface of the plates and the chord.

On the other side, at the bottom surface of the body, the stagnation point moves downstream as the angle of attack increases, giving more opportunity to the flow to reattach. The recirculation bubble has again enough energy to provoke galloping when disappears, but now the bubble is in the leading edge of the aerofoil. The characteristic length available for the bubble to reattach changes from the chord to the thickness of the aerofoil as the aspect ratio increases.

The evolution of the recirculation bubble can be observed in Fig. 8 showing the pressure distributions measured on the rhombus with $\tau=0.36$ at different angles of attack. At $\alpha=5^{\circ}$ there is a small suction peak close to the leading edge at the upper surface, whereas at the lower surface results show the existence of a stagnation point close to the windward wedge. The suction peak corresponds to a recirculation bubble where, as explained above, the fluid recirculates at high speed thus causing the decrease of the measured pressure [23]. As the angle of attack grows the recirculation bubble becomes larger, the magnitude of the suction peak increases and the bubble reattachment point moves leeward (at $\alpha=10^{\circ}$ the recirculation bubble extends from the leading edge to a point located at $x / c \simeq 0.2$, Fig. 8). The bubble reattachment point moves through the trailing edge with the angle of attack, so that at $\alpha=15^{\circ}$ the recirculation bubble ends close to $x / c \cong 0.3$. Note also that at this angle of attack the lower surface also shows a long recirculation bubble which is formed after the boundary layer separation at $x / c=0.5$ and the subsequent reattachment at $x / c \cong 0.9$.

At $\alpha=20^{\circ}$ the reattachment point of the upper leading edge recirculation bubble reaches the middle wedge of the rhombus, whereas in the lower surface the corresponding bubble length is shortened because the second flat surface of the lower side becomes more and more vertical, thus favoring the reattachment of the detached lower-side boundary layer. Such a behavior is even strengthened when the angle of attack increases further, in such a way that at $\alpha=30^{\circ}$ the whole boundary layer at the upper surface becomes separated, whereas the recirculation bubble at the lower surface ends at $x / c \cong 0.7$.

All these results are summarized in Fig. 9, where the variation with the angle of attack of both the lift coefficient and the drag coefficient are represented. Note that within the range of angles of attack under consideration the drag coefficient continuously grows while the lift slope curve is positive, $\mathrm{dc} / \mathrm{d} \alpha>0$, in the whole $\alpha$ range. Therefore, since both $c_{d}$ and $\mathrm{d}_{l} / \mathrm{d} \alpha$ are positive, the Den Hartog criterion for galloping instability cannot be fulfilled in the $\alpha$ interval under consideration.

\section{Conclusions}

The transverse galloping of rhombic bodies have been analyzed by means of dynamic wind tunnel tests (test models elastically supported and allowed to oscillate freely). Different geometries varying the relative thickness of the rhombi have been investigated to understand the dependence of the galloping instability with this geometrical parameter. A remarkable result is that the rhombus geometry, which is unstable for a wide range of values of the relative thickness, presents stability in a small region between $\tau \cong 0.36$ and $\tau \cong 0.40$. Triangular profiles have also been analyzed to compare their unstable behavior with the stability shown by rhombi. The suitability of the quasi-static criterion for this type of bodies in the tested conditions (mechanical properties and turbulence of the incoming flow) has been verified. 
Since galloping instability is related to the flow morphology around the body, another set of wind tunnel tests has been performed on the rhombic cross section to measure the pressure distribution on the body surfaces. From those measurements it was concluded that the rhombi stability (within the defined range of relative thickness $0.36<\tau<0.40$ ) at small angles of attack comes from the recirculation bubble formed at the upper surfaces of the cross section, whose smaller size compared to the thinner aerofoils $(\tau<0.36)$ prevents the lift coefficient from dropping with the same intensity when the angle of attack is increased. For larger values of the angle of attack, the boundary layer is completely separated at the body's upper surface; this fact, together with the particular geometry of rhombi, makes the lift coefficient curves slope positive and therefore causes galloping stability. On the other hand, in the rhombi with higher thickness $(\tau>0.40)$ the stagnation point moves downstream in the lower surface, the characteristic length available for the bubble to reattach varies as the thickness increases, from the chord to the thickness, allowing the drop in the lift to appear again with enough intensity for galloping when the bubble disappears.

\section{Acknowledgments}

Partial support to this work from the Consejo Nacional de Ciencia y Tecnología of Mexico through their scholarships program to the first author is acknowledged.

\section{References}

[1] P. McComber, A. Paradis, A cable galloping model for thin ice accretions, Atmospheric Research 46 (1998) 13-25.

[2] O. Chabart, J.L. Lilien, Galloping of electrical lines in wind tunnel facilities, Joumal of Wind Engineering and Industrial Aerodynamics 74-76 (1998) 967-976.

[3] Q. Zhang, N. Popplewell, A.H. Shah, Galloping of bundle conductor, Journal of Sound and Vibration 234 (2000) 115-134.

[4] N. Pulipaka, P.P. Sarkar, J.R. McDonald, On galloping vibration of traffic signal structures, Journal of Wind Engineering and Industrial Aerodynamics 77 and 78 (1998) 327-336.

[5] J.A. Rice, D.A. Foutch, J.M. LaFave, S. Valdovinos, Field testing and analysis of aluminum highway sign trusses, Engineering Structures 34 (2012) $173-186$.

[6] K.W. Johns, R.J. Dexter, The development of fatigue design load ranges for cantilevered sign and signal support structures, Journal of Wind Engineering and Industrial Aerodynamics 77\&78 (1998) 315-326.

[7] D. St. Clair, A. Bibo, V.R. Sennakesavababu, M.F. Daqaq, G. Li, A scalable concept for micropower generation using flow-induced self-excited oscillations, Applied Physics Letters 96 (2010) 144103, http://dx.doi.org/10.1063/1.3385780. (3 pp).

[8] J. Peng, G.S. Chen, Flow-oscillating structure interactions and the applications to propulsion and energy harvest, Applied Physics Research ;4http://dx. doi.org/10.5539/apr.v4n2p1. (14 pp).

[9] Y.T. Ng, S.C. Luo, Y.T. Chew, On using high-order polynomial curve fits in the quasi-steady theory for square-cylinder galloping, Journal of Fluids and Structures 20 (2005) 141-146.

[10] X. Wu, F. Ge, Y. Hong, A review of recent studies on vortex-induced vibrations of long slender cylinders, Journal of Fluids and Structures 28 (2012) 292-308.

[11] A. Barrero-Gil, G. Alonso, A. Sanz-Andrés, Energy harvesting from transverse galloping, Journal of Sound and Vibration 329 (2010) $2873-2883$.

[12] H.J. Jung, S.W. Lee, D.D. Jang, Feasibility study on a new energy harvesting electromagnetic device using aerodynamic instability, IEEE Transactions on Magnetics 45 (2009) 4376-4379.

[13] H.J. Jung, S.W. Lee, The experimental validation of a new energy harvesting system based on the wake galloping phenomenon, Smart Materials and Structures 20 (2011) 055022, http://dx.doi.org/10.1088/0964-1726/20/5/055022. (10 pp).

[14] H.D. Akaydin, N. Elvin, Y. Andreopoulos, Wake of a cylinder: a paradigm for energy harvesting with piezoelectric materials, Experiments in Fiuids 49 (2010) 291-304.

[15] G. Alonso, J. Meseguer, I. Pérez-Grande, Galloping oscillations of two-dimensional triangular cross-sectional bodies, Experiments in Fluids 38 (2005) 789-795.

[16] G. Alonso, J. Meseguer, A. Sanz-Andrés, E. Valero, On the galloping instability of two-dimensional bodies having elliptical cross sections, Journal of Wind Engineering and Industrial Aerodynamics 98 (2010) 438-448.

[17] G. Alonso, E. Valero, J. Meseguer, An analysis on the dependence on cross section geometry of galloping stability of two-dimensional bodies having either biconvex or rhomboidal cross sections, European Journal of Mechanics-B Fhids 28 (2009) 328-334.

[18] P. Hémon, F. Santi, On the aeroelastic behaviour of rectangular cylinders in cross-flow, Journal of Fluids and Structures 16 (7) (2002) 855-889.

[19] E. Dragomirescu, H. Yamada, H Katsuchi, Experimental investigation of the aerodynamic stability of the "Endless Column", Romania, Joumal of Wind Engineering and Industrial Aerodynamics 97 (2009) 475-484.

[20] J.P. Den Hartog, Mechanical Vibrations, 4th ed. McGraw-Hill, New York, 1956.

[21] I.H. Abbott, A.E. Von Doenhoff, Theory of Wing Sections, Dover Publications lnc., New York, 1959.

[22] G. Alonso, A. Sanz-Lobera, J. Meseguer, Hysteresis phenomena in transverse galloping of triangular cross-section bodies, Journal of Fluids and Structures 33 (2012) 243-251.

[23] G. Alonso, J. Meseguer, A parametric study of the galloping stability of two-dimensional triangular cross-section bodies, Journal of Wind Engineering and Industrial Aerodynamics 94 (2006) 241-253. 